\title{
IDENTIFICAÇÃO DE CONTEÚDOS DE QUÍMICA COMO PRÉ-REQUISITOS PARA AS DISCIPLINAS ESPECÍFICAS DO CURSO TÉCNICO EM FARMÁCIA
}

\author{
Alessandra Lobo da Silva ROSA ${ }^{1,2,3}$, Aldo SHIMOYA², Eduardo SHIMODA², Fabrício Ferreira de \\ Albuquerque FERNANDES ${ }^{4}$, Erik da Silva OLIVEIRA ${ }^{2}$, Gabriella Lima Viana CHAGAS ${ }^{1,2}$, Nathália Ramos \\ Ribeiro $\operatorname{ROSA}^{2}$ \& Henrique Rego Monteiro DA HORA ${ }^{4}$ \\ 1 Serviço Nacional de Aprendizagem Comercial. Campos dos Goytacazes, Rio de Janeiro, Brasil. \\ 2 Universidade Candido Mendes. Campos dos Goytacazes, Rio de Janeiro, Brasil. \\ 3 Universidade Estácio de Sá - Campos dos Goytacazes, Rio de Janeiro, Brasil. \\ 4 Instituto Federal de Educação, Ciência e Tecnologia Fluminense. Campos dos Goytacazes, Rio de Janeiro, Brasil. \\ *Autor para correspondência: shimoda@ucam-campos.br
}

DOI: http://dx.doi.org/10.18571/acbm.138

\section{RESUMO}

Uma das bases de um curso de farmácia é a química, sendo que uma gama de conteúdo é incluída na ementa da disciplina. No entanto, muitas vezes, torna-se difícil o cumprimento de todos os conteúdos. É importante, portanto, a identificação de conteúdos que são essenciais diante da restrição de tempo existente em alguns casos. Neste contexto, o objetivo do presente trabalho foi identificar os conteúdos de química que são essenciais para as disciplinas específicas do curso técnico em farmácia. Foi elaborado e aplicado um questionário contendo dimensões e conteúdos da disciplina de química. A coleta de dados se deu entre maio e julho de 2015, entrevistando-se 84 alunos no último módulo e 11 docentes de cursos técnicos de farmácia em Campos dos Goytacazes, RJ. Foi aplicado o método de Lawshe para validação dos itens. Como resultado, percebeu-se que a maioria dos conteúdos das dimensões: "tabela periódica", "fundamentos das reações químicas", "misturas e soluções", "acidez e basicidade" e "funções inorgânicas" foram consideradas como essenciais, tanto sob a percepção de docentes quanto dos discentes. No caso das dimensões: "átomos", "ligação química", "moléculas" e "química orgânica", há necessidade de reavaliação, aumentando-se o tamanho da amostra, para conclusões mais definitivas. Concluiu-se que parte dos conteúdos de química disponibilizados para o curso técnico em farmácia poderiam ser excluídos, sugerindo-se uma reavaliação e discussão das ementas, o que permitiria otimizar o tempo e investimento de esforços em conteúdos realmente prioritários para a formação técnica e o exercício da profissão.

Palavras chave: Química; Farmácia; Lawshe; Questionário.

\begin{abstract}
One of the bases of a course in pharmacy is chemistry, and a range of content is included in the menu of the discipline. However, often it is difficult to comply with all the contents. It is important, therefore, identification of content which are essential before the existing time constraints in some cases. In this context, the objective of this study was to identify the chemical contents that are essential to the specific disciplines of technical course and pharmacy. It was developed and implemented a questionnaire containing dimensions and content of the discipline of chemistry. Data collection took place between May and July 2015, was interviewing 84 students in the last module and 11 teachers of technical courses in pharmacy in Campos dos Goytacazes. Was applied Lawshe method for validation of the items. As a result, it was noticed that most of the dimensions of the content: "periodic table", "fundamentals of chemical reactions", "mixtures and solutions", "acidity and alkalinity" and "inorganic functions" were considered as essential, both in the perception of teachers as the students. In the case of
\end{abstract}




\section{Biomedica Brasiliensia}

dimensions, "atoms", "chemical bond", "molecules" and "organic chemistry", no need to reevaluation by increasing the sample size to more definitive conclusions. It was concluded that some of the chemical content available to the technical course in pharmacy could be excluded, suggesting a review and discussion of the menus, which would optimize the time and investment efforts in really priority content for technique and the profession.

Keywords: Chemistry; Drugstore; Lawshe; Questionnaire.

\section{Introdução}

A Química surgiu como uma disciplina unicamente experimental, servindo como base para explicar numerosos fenômenos existentes na natureza. No processo de evolução da Química, são mencionados os experimentos dos alquimistas os quais almejavam a vida eterna utilizando misturas de várias substâncias, cujo objetivo era a obtenção da pedra filosofal. Suas pesquisas foram fundamentais para os diversos conhecimentos alcançados e usados na ciência atualmente (VITORINO et al., 2009).

Na prática docente, é constante o questionamento por parte dos alunos acerca do motivo pelo qual estudam química em razão de que nem sempre este conhecimento será necessário na futura profissão. O conhecimento da química se deve basicamente ao fato de proporcionar ao homem o desenvolvimento de uma visão crítica do mundo que o cerca, podendo verificar, compreender e utilizar este conhecimento no seu dia a dia, tendo condições de investigar e interceder em situações que contribuem para a deterioração de sua qualidade de vida (CARDOSO, 2000). Assim, a aprendizagem pode ser verificada através de uma ação motivada, da sistematização de uma situação. Aprender é uma prática de conhecimento da realidade concreta, isto é, da situação real vivenciada pelo aluno, que se dá através de uma aproximação criteriosa dessa realidade (LOPES et al., 2015). O objetivo desse estudo foi identificar os conteúdos de química que são essenciais para as disciplinas específicas do curso técnico de farmácia.

\section{Revisão Bibliográfica}

\subsection{Ensino Técnico de Farmácia no Brasil}

A educação profissional e tecnológica assume valor estratégico para o desenvolvimento nacional resultante das transformações ao longo das últimas décadas com a criação dos Institutos Federais de Educação, Ciência e Tecnologia e com a intensificação e diversificação das atividades de ensino visando a atender os mais diferenciados públicos nas modalidades: presencial, semipresencial e a distância (BERGER FILHO, 1999).

De acordo com o Instituto Nacional de Estudos Pedagógicos Anísio Teixeira (INEP/MEC), entre os cursos técnicos da educação profissional, a área da saúde congrega o maior número de alunos e tem apresentado crescimento progressivo nesse quantitativo. $\mathrm{O}$ ensino profissional demanda não apenas as atribuições operacionais, assim como a compreensão global do processo produtivo, com a percepção do saber tecnológico, a valorização da cultura do trabalho e a motivação de atitudes necessárias à tomada de decisões (GRECO, 2009). De acordo com o Catálogo Nacional de Cursos Técnicos, emitido pelo Ministério da Educação em 2009, o técnico em farmácia é o profissional que desempenha operações farmacotécnicas, identificando e classificando os diferentes tipos de produtos e de formas farmacêuticas, sua composição e técnica de preparação (BRASIL, 2009). Entre os maiores consumidores mundiais de medicamentos encontra-se o Brasil, sendo, portanto, um fator preocupante quanto à saúde 
pública. Dessa forma, o sucesso terapêutico no tratamento de doenças dependerá de bases que permitam a escolha do tratamento medicamentoso, a seleção do medicamento de forma científica e racional, considerando sua efetividade, segurança e custo, e ainda uma dispensação em condições adequadas. As relações estabelecidas entre os profissionais de farmácia e o usuário são relevantes para a efetividade terapêutica (BRASIL, 2012).

A área de Farmácia envolve resultados relacionados à produção, dispensação e comercialização de medicamentos alopáticos e homeopáticos, cosméticos e fitoterápicos. O processo de produção na área de Farmácia está dividido em duas dimensões essenciais de atuação que são a dispensação de produtos farmacêuticos (incluindo o atendimento e a venda), assim como a produção de medicamentos e cosméticos (PPC SENAC, 2013). Nesse contexto, a amplificação do ramo farmacêutico tem sido complementada pela crescente demanda por profissionais qualificados e bem preparados. $\mathrm{O}$ curso Técnico em Farmácia objetiva atender a necessidade de informações pertinentes para aqueles que almejam trabalhar em estabelecimentos farmacêuticos, seja no setor administrativo, comercial ou de produção. Para matrícula na Habilitação Técnica de Nível Médio será exigido, no mínimo, estar cursando o $2^{\circ}$ ano do Ensino Médio (PPC SENAC, 2013).

\subsection{Ensino da Química}

Na medida em que a Ciência e a Tecnologia tornaram-se primordiais para a evolução humana em vários aspectos como o aspecto científico, social, econômico e cultural, o ensino de ciências também foi crescendo e organizando-se num conjunto suscetível a várias transformações (LIMA; LEITE, 2012). A química é uma ciência que está inserida no programa curricular do ensino fundamental, médio e técnico. O estudo da química deve viabilizar aos alunos a percepção das transformações químicas de modo integral para que os mesmos possam associar as informações adquiridas e tomar decisões promovendo interação com o universo enquanto indivíduos (ALMEIDA et al, 2013). O tradicionalismo ainda pode ser observado na metodologia do Ensino de química na educação básica, ressaltando-se as técnicas de memorização de regras, fórmulas, nomes e estruturas, aplicação de conteúdos completamente distanciados da atualidade, podendo ocasionar desmotivação e desinteresse por parte dos alunos (LIMA; LEITE, 2012). As avaliações realizadas pelo Exame Nacional do Ensino Médio (ENEM) revelam que alguns alunos não produzem respostas adequadas a partir de um conjunto de dados que exigem interpretação, leitura de tabelas, quadros e gráficos, e não conseguem fazer comparações ou fundamentar seus julgamentos (BRASIL, 2006). Uma estratégia para facilitar o ensino e ajudar no entendimento dos conteúdos de química é a aula prática. As aulas práticas auxiliam a compreensão da ciência da natureza e despertam no aluno o interesse pela química. Assim, contextualizar os conteúdos contribui para o fator motivacional e para a construção do conhecimento de uma forma holística. A contextualização surge nas diretrizes que estão definidas nos Parâmetros Curriculares Nacionais (PCNs), os quais visam um ensino de química centrado na interface entre informação científica e contexto social (ALMEIDA et al, 2008).

Segundo Almeida et al. (2008), uma das funções do ensino da química é reconhecer a importância da ciência na busca do aprendizado. Na área de Farmácia, essa disciplina auxilia na compreensão de vários fatores, como por exemplo, estruturas dos medicamentos, suas ligações e interações com diferentes sítios no organismo.

\subsection{Validação dos Itens do Questionário}

O método de Lawshe é um dos métodos mais antigo e também muito utilizado para quantificar a validade de conteúdos (WILSON; PAN; SCHUMSKY, 2012). O método é embasado na aplicação de questionários a especialistas que avaliam um item do questionário 
como 1- essencial, 2- importante, mas não essencial e 3- não importante e N- não sei/não quero opinar. Em seguida, agrupam-se as respostas como essenciais ou não essenciais e, fundamentado em uma distribuição binomial, verifica-se a validade do item (DANTIER; MIRANDA, 2014). No método elaborado por Lawshe para cada item do questionário uma taxa de conteúdo é calculada, é conhecido em inglês por Content Validity Ratio (CVR). O CVR é calculado através da fórmula seguinte:

$$
\mathrm{CVR}=\frac{\mathrm{n}_{\mathrm{e}}-(\mathrm{N} / 2)}{(\mathrm{N} / 2)}
$$

Onde: " $\mathrm{n}_{\mathrm{e}}$ " refere-se ao número de especialistas que classificaram cada item como "essencial", e "N" refere-se número total de especialistas que analisam os itens.

O método de Lawshe foi utilizado em vários trabalhos na área médica, dentre eles: controle de sintomas pré-menstruais (HARIRI et al., 2013), fatores de risco do sobrepeso e obesidade (GHAVAMZADEH et al., 2013), impactos psicossociais (ORELLANO; JUTAI, 2013), gastos com a saúde relacionados a pacientes portadores de doença renal crônica (DEVRAJ; WALLACE, 2013), oncologia pediátrica (REEVE et al. 2013), escala de osteoporose para pacientes com diabetes (ABDULAMEE et al., 2013), adolescentes com problemas de saúde e as medidas a serem tomadas (BAHEIRAEI et al., 2013), disfunção sexual feminina (MAASOUMI et al., 2013), perfil e usabilidade de ambulatório de saúde (DRUM et al., 2012), sensações provocadas por agulhas na acupuntura (YU; JONES; PANG, 2012), ovários policísticos (BAZARGANIPOUR et al., 2012). Wilson, Pan e Schumsky (2012), no entanto revisaram os cálculos propostos por Lawshe e estabeleceram uma nova tabela, corrigindo a tabela original. Diante disso, Ayre e Scally (2014) discutiram os artigos de Lawshe (1975), Wilson, Pan e Schumsky (2012), propondo métodos para cálculo inicial de valores críticos e tabelas de probabilidades binomial exatas (Figura 1).

\begin{tabular}{ccccccc}
\hline & \multicolumn{6}{c}{ Teste de significância unicaudal } \\
\cline { 2 - 7 } & $10 \%$ & $5 \%$ & $2,50 \%$ & $1 \%$ & $0,50 \%$ & $0,10 \%$ \\
\cline { 2 - 7 } Número de & \multicolumn{6}{c}{ Teste de Significância bicaudal } \\
\hline Especialistas & $20 \%$ & $10 \%$ & $5 \%$ & $2 \%$ & $1 \%$ & $0,20 \%$ \\
\hline 5 & 0,573 & 0,736 & 0,877 & 0,990 & 0,990 & 0,990 \\
6 & 0,523 & 0,672 & 0,800 & 0,95 & 0,990 & 0,990 \\
7 & 0,484 & 0,622 & 0,741 & 0,879 & 0,974 & 0,990 \\
8 & 0,453 & 0,582 & 0,693 & 0,822 & 0,911 & 0,990 \\
9 & 0,427 & 0,548 & 0,653 & 0,775 & 0,859 & 0.990 \\
10 & 0,405 & 0,520 & 0,620 & 0,736 & 0,815 & 0,977 \\
11 & 0,386 & 0,496 & 0,591 & 0,701 & 0,777 & 0,932 \\
12 & 0,370 & 0,475 & 0,566 & 0,672 & 0,744 & 0,892 \\
13 & 0,355 & 0,456 & 0,544 & 0,645 & 0,714 & 0,857 \\
14 & 0,343 & 0,440 & 0,524 & 0,622 & 0,688 & 0,826 \\
15 & 0,331 & 0,425 & 0,506 & 0,601 & 0,665 & 0,798 \\
16 & 0,320 & 0,411 & 0,490 & 0,582 & 0,644 & 0,773 \\
17 & 0,311 & 0,399 & 0,475 & 0,564 & 0,625 & 0,749 \\
18 & 0,302 & 0,388 & 0,462 & 0,548 & 0,607 & 0,728 \\
19 & 0,294 & 0,377 & 0,450 & 0,534 & 0,591 & 0,709 \\
20 & 0,287 & 0,368 & 0,438 & 0,520 & 0,576 & 0,691 \\
\hline
\end{tabular}


Figura 1: CVRcrítico (mínimo) por número de especialistas para cada item. Fonte: Adaptado de Wilson, Pan e Schumsky (2012).

\section{Material e Métodos}

Inicialmente, foram selecionados itens da dimensão "conteúdo programático de química" do Projeto Pedagógico do Curso Técnico de nível médio em Farmácia de uma Instituição de ensino para elaboração do questionário. Foi excluída a dimensão fundamentos de química e os itens relacionados, apresentação do curso, a química e a sociedade, os ramos da química e a importância da Química para o técnico em farmácia.

Em seguida, foram entrevistados, durante os meses de maio, junho e julho de 2015, 84 alunos que cursavam o último módulo do curso técnico em farmácia, sendo oferecidas as seguintes opções de resposta para cada item: (1) não importante; (2) importante, mas não essencial; (3) essencial e (N) não sei. A escala de percepção utilizada foi a de Lawshe (1975). As dimensões analisadas foram: 1) Átomos; 2) Propriedades periódicas; 3) Ligações químicas; 4) Moléculas; 5) Fundamentos das reações químicas; 6) Misturas e soluções; 7) Acidez e basicidade; 8) Funções inorgânicas e 9) Química orgânica. Posteriormente, os questionários foram aplicados a 11 professores das disciplinas de química e/ou específica do curso de farmácia, de instituições públicas e particulares da cidade de Campos dos Goytacazes, durante o mês de julho de 2015. Foi obtida, então, a frequência relativa (\%) de respondentes que consideravam o item como essencial (FRessencial) através da razão entre os que assinalaram a opção "(3) essencial” e o total de entrevistados, excluindo-se destes os que responderam "(N) não sei. A FR essencial apresenta valores que podem variar de $0 \%$ a $100 \%$. Foi calculado, então, para cada item, o "Content Validity Ratio" (CVR) interpolando-se a FR essencial para valores de (1) a $(+1)$.

Em seguida, foi calculado o valor mínimo do CVR (CVR crítico) para que o item seja incluído no questionário. Como as respostas foram agrupadas como dicotômicas (essencial ou não essencial), estas seguem uma distribuição binomial, com possibilidade de aproximação para distribuição normal, com média $\mu=n$.p e variância $\sigma^{2}=n . p .(1-p)$ sendo $n=$ número de respondentes (excluindo-se os que assinalaram "não sei") e p= probabilidade de ocorrência como essencial, no qual adotou-se o valor igual a 0,5. Utilizando-se, o nível de significância de 5\%, foi calculado o CVR crítico. Para análise dos dados apresentados a partir dos questionários respondidos, uma avaliação estatística descritiva foi efetuada, considerando a frequência e o grau de importância (médias) nas respostas. O método de Lawshe (1975) foi utilizado para verificar a validade dos itens.

\section{Resultados e Discussão}

\section{1 Átomos}

Na Tabela 1 são apresentados os cálculos relacionados à validação dos itens na dimensão "átomos".

Tabela 1: Resultados do método de Lawshe para a dimensão "átomos".

\begin{tabular}{|c|c|c|c|c|c|c|c|}
\hline Item & Estrato & $\mathrm{Ne}$ & $\mathrm{N}$ & $\% \mathrm{Ne}$ & CVRcalc & CVRtab & Decisão \\
\hline \multirow{2}{*}{2.1 - Conceito Geral } & Alunos & 40 & 80 & $50,0 \%$ & 0,000 & 0,219 & Reavaliar \\
\hline & Docentes & 7 & 11 & $63,6 \%$ & 0,273 & 0,591 & Reavaliar \\
\hline \multirow{2}{*}{ 2.2 - Modelos atômicos } & Alunos & 33 & 80 & $41,3 \%$ & $-0,175$ & 0,219 & Reavaliar \\
\hline & Docentes & 4 & 11 & $36,4 \%$ & $-0,273$ & 0,591 & Reavaliar \\
\hline \multirow{2}{*}{$\begin{array}{l}2.3 \text { - Números quânticos e orbitais } \\
\text { atômicos }\end{array}$} & Alunos & 25 & 75 & $33,3 \%$ & $-0,333$ & 0,226 & Excluir \\
\hline & Docentes & 2 & 11 & $18,2 \%$ & $-0,636$ & 0,591 & Excluir \\
\hline \multirow{2}{*}{2.4 - Distribuição eletrônica } & Alunos & 46 & 81 & $56,8 \%$ & 0,136 & 0,218 & Reavaliar \\
\hline & Docentes & 6 & 11 & $54,5 \%$ & 0,091 & 0,591 & Reavaliar \\
\hline
\end{tabular}


É possível perceber, pela Tabela 1, que os todos os itens, exceto o item 2.3. Números quânticos e orbitais atômicos foram classificados como "reavaliação", tanto na opinião de alunos quanto docentes. Observa-se que o item "2.3. Números quânticos e orbitais atômicos" foi classificado pelos alunos e docentes como "excluir", tendo em vista que o CVR calculado foi menor do que o tabelado. Quanto ao item "2.3. Números quânticos e orbitais atômicos"; 43,4\% dos alunos e 54,5\% dos docentes acham o item importante, mas não essencial. Verifica-se também que $16,9 \%$ dos alunos e $27,3 \%$ dos docentes acham que o item não é importante. Quanto ao grau de importância, os alunos avaliaram este item com média equivalente a 2,15; enquanto os docentes analisaram o mesmo com média correspondente a 1,91. Provavelmente, a exclusão do item "2.3 Números quânticos e orbitais atômicos" tanto na percepção de alunos quanto na dos docentes, se deve ao fato deste assunto não se contextualizar diretamente com as disciplinas da farmácia. De acordo com Penning et al. (2013), diante dos desafios encontrados na educação em química, torna-se imprescindível a contribuição para uma melhor abordagem aos assuntos debatidos em sala de aula, utilizando a realidade para um melhor entendimento do aluno e contextualizando com as necessidades e interesses da comunidade na qual a escola está inserida.

\subsection{Propriedades Periódicas}

Na Tabela 2 são apresentados os cálculos relacionados à validação dos itens na dimensão "Propriedades periódicas".

Tabela 2: Resultados do método de Lawshe para a dimensão "Propriedades periódicas".

\begin{tabular}{|c|c|c|c|c|c|c|c|}
\hline Item & Estrato & $\mathrm{Ne}$ & $\mathrm{N}$ & $\% \mathrm{Ne}$ & CVRcalc & CVRtab & Decisão \\
\hline \multirow{2}{*}{3.1 - A tabela periódica } & Alunos & 69 & 82 & $84,1 \%$ & 0,683 & 0,216 & Manter \\
\hline & Docentes & 10 & 11 & $90,9 \%$ & 0,818 & 0,591 & Manter \\
\hline \multirow{2}{*}{$\begin{array}{l}3.2 \text { - Classificação dos elementos } \\
\text { químicos }\end{array}$} & Alunos & 63 & 81 & $77,8 \%$ & 0,556 & 0,218 & Manter \\
\hline & Docentes & 10 & 11 & $90,9 \%$ & 0,818 & 91 & Manter \\
\hline \multirow{2}{*}{$\begin{array}{l}3.3 \text { - Números Quânticos e orbitais } \\
\text { atômicos }\end{array}$} & Alunos & 32 & 72 & $44,4 \%$ & $-0,111$ & 0,231 & Reavaliar \\
\hline & Docentes & 2 & 11 & $18,2 \%$ & $-0,636$ & 0,591 & Excluir \\
\hline
\end{tabular}

De acordo com a Tabela 2, observa-se que os itens "3.1 A tabela periódica" e "3.2 Classificação dos elementos químicos" foram classificados como "Manter" na opinião dos alunos e docentes. Verifica-se, que $83,1 \%$ dos alunos e $90,9 \%$ dos docentes avaliaram o item "3.1 A tabela periódica" como "Essencial". Também é possível verificar que 75,9\% dos alunos e 90,9\% dos docentes avaliaram o item "3.2 Classificação dos elementos químicos" como "Essencial". Quanto ao grau de importância, os alunos e os docentes avaliaram o item "3.1 A tabela periódica" com médias equivalentes a 2,80 e 2,82 respectivamente. Já em relação ao item "3.2 Classificação dos elementos químicos", os alunos e docentes avaliaram com médias correspondentes a 2,75 e 2,82 respectivamente. Observa-se, de acordo com a tabela 2, que o item "3.3 Números Quânticos e orbitais atômicos" foi classificado como "Reavaliar" pelos alunos, e "Excluir" pelos docentes.

Quanto ao grau de importância, os alunos avaliaram este item com média equivalente a 2,29; enquanto os docentes analisaram o mesmo com média correspondente a 1,82. Considerando a importância do estudo da química para as disciplinas específicas do curso de farmácia, os itens "A tabela periódica" e "elementos químicos" foram mantidos tanto na percepção dos alunos quanto na dos docentes.

Observa-se que estes itens possuem uma influência direta nas disciplinas específicas de Farmacologia, Farmacotécnica e Farmácia Hospitalar a serem estudados no curso de farmácia, visto que alguns elementos da tabela periódica fazem parte da composição e preparação dos medicamentos. 


\subsection{Ligações Químicas}

Na Tabela 3 são apresentados os cálculos relacionados à validação dos itens na dimensão "Ligações químicas".

Tabela 3: Resultados do método de Lawshe para a dimensão "Ligações químicas".

\begin{tabular}{|c|c|c|c|c|c|c|c|}
\hline Item & Estrato & $\mathrm{Ne}$ & $\mathrm{N}$ & $\% \mathrm{Ne}$ & CVRcalc & CVRtab & Decisão \\
\hline \multirow{2}{*}{4.1 - Ligação Iônica } & Alunos & 55 & 82 & $67,1 \%$ & 0,341 & 0,216 & Manter \\
\hline & Docentes & 8 & 11 & $72,7 \%$ & 0,455 & 0,591 & Reavaliar \\
\hline \multirow{2}{*}{4.2 - Ligação Covalente } & Alunos & 55 & 82 & $67,1 \%$ & 0,341 & 0,216 & Manter \\
\hline & Docentes & 7 & 11 & $63,6 \%$ & 0,273 & 0,591 & Reavaliar \\
\hline \multirow{2}{*}{4.3 - Estruturas de lewis } & Alunos & 38 & 78 & $48,7 \%$ & $-0,026$ & 0,222 & Reavaliar \\
\hline & Docentes & 5 & 11 & $45,5 \%$ & $-0,091$ & 0,591 & Reavaliar \\
\hline \multirow{2}{*}{4.4 - Carga Formal } & Alunos & 22 & 62 & $35,5 \%$ & $-0,290$ & 0,249 & Excluir \\
\hline & Docentes & 5 & 11 & $45,5 \%$ & $-0,091$ & 0,591 & Reavaliar \\
\hline \multirow{2}{*}{4.5 - Estrutura de Ressonância } & Alunos & 19 & 61 & $31,1 \%$ & $-0,377$ & 0,251 & Excluir \\
\hline & Docentes & 2 & 11 & $18,2 \%$ & $-0,636$ & 0,591 & Excluir \\
\hline \multirow{2}{*}{4.6 - Forças Intermoleculares } & Alunos & 28 & 71 & $39,4 \%$ & $-0,211$ & 0,233 & Reavaliar \\
\hline & Docentes & 6 & 11 & $54,5 \%$ & 0,091 & 0,591 & Reavaliar \\
\hline
\end{tabular}

De acordo com a Tabela 3, observa-se que o único item que foi classificado como "Excluir" na opinião dos alunos e docentes foi o "4.5 Estrutura de Ressonância". Observa-se também que $36,1 \%$ dos alunos e $63,6 \%$ dos docentes avaliaram este item como "Importante, mas não essencial". Quanto ao grau de importância, verifica-se que os alunos e docentes avaliaram este item com médias correspondentes a 2,11 e 2,00 respectivamente. É possível observar, de acordo com a tabela 3, que itens como "4.3 Estruturas de Lewis" e "4.6 Forças Intermoleculares" foram classificados como "Reavaliar" tanto por alunos, quanto por docentes. Também se observa que o item "4.4 Carga Formal" foi classificado como "Excluir" pelos alunos, em contrapartida avaliado como "Reavaliar" pelos docentes.

Verifica-se que itens como "4.1 Ligação Iônica" e "4.2 Ligação covalente" foram classificados como "Manter" pelos alunos e "Reavaliar" pelos professores (Tabela 3). Quanto ao grau de importância do item "4.1 Ligação Iônica", que os alunos avaliaram este com média equivalente a 2,62; e os docentes com média correspondente a 2,64. Em relação ao grau de importância do item "4.2 Ligação covalente", este foi avaliado pelos alunos e docentes com médias correspondentes a 2,62 e 2,55 respectivamente.

Conforme a percepção dos alunos, o item " 4.4 carga formal” deverá ser excluído, provavelmente por ser um conteúdo em que a maior parte dos alunos encontra dificuldades de compreensão, visto que, para a determinação da carga formal de um átomo é necessário trabalhar com expressões e cálculos interligados a estrutura eletrônica de Lewis. De acordo com os PCNs para o ensino da química, o Exame Nacional do Ensino Médio (ENEM), mostra que os alunos não têm conseguido produzir respostas coerentes a partir de um conjunto de dados que exigem interpretação e não conseguem fazer comparações ou fundamentar seus entendimentos.

Tratando-se do "item 4.5 estrutura de ressonância" que em química corresponde a uma ferramenta bastante empregada na orgânica, deverá ser excluído tanto na percepção de alunos quanto na de docentes provavelmente por se tratar de um assunto de difícil contextualização para os docentes e com grau de complexidade para o entendimento e pouca aplicabilidade para as disciplinas específicas do curso Técnico em Farmácia. Almeida et al. (2008) relatam em seu trabalho que as contextualizações dos conteúdos são de extrema importância, como fator motivacional e para a construção do conhecimento de uma forma holística. 


\subsection{Moléculas}

Na Tabela 4 são apresentados os cálculos relacionados à validação dos itens na dimensão "Moléculas".

Tabela 4: Resultados do método de Lawshe para a dimensão "Moléculas".

\begin{tabular}{llcccccc}
\hline Item & Estrato & $\mathrm{Ne}$ & $\mathrm{N}$ & $\% \mathrm{Ne}$ & CVRcalc & CVRtab & Decisão \\
\hline \multirow{2}{*}{5.1 - Forma das moléculas e dos íons } & Alunos & 35 & 77 & $45,5 \%$ & $-0,091$ & 0,223 & Reavaliar \\
\cline { 2 - 8 } & Docentes & 7 & 11 & $63,6 \%$ & 0,273 & 0,591 & Reavaliar \\
\hline \multirow{2}{*}{5.2 - Modelo VSEPR } & Alunos & 16 & 48 & $33,3 \%$ & $-0,333$ & 0,283 & Excluir \\
\cline { 2 - 8 } & Docentes & 1 & 7 & $14,3 \%$ & $-0,714$ & 0,741 & Reavaliar \\
\hline \multirow{2}{*}{5.3 - Polaridade das moléculas } & Alunos & 35 & 69 & $50,7 \%$ & 0,014 & 0,236 & Reavaliar \\
\cline { 2 - 8 } & Docentes & 7 & 10 & $70,0 \%$ & 0,400 & 0,620 & Reavaliar \\
\hline \multirow{2}{*}{5.4 - Hibridização dos orbitais } & Alunos & 14 & 55 & $25,5 \%$ & $-0,491$ & 0,264 & Excluir \\
\cline { 2 - 8 } & Docentes & 3 & 10 & $30,0 \%$ & $-0,400$ & 0,620 & Reavaliar \\
\hline
\end{tabular}

É possível perceber, pela Tabela 4, que os itens "5.1 Forma das moléculas e dos íons" e o "5.3 Polaridade das moléculas", foram classificados como "Reavaliar" tanto para os alunos, quanto para os docentes. Quanto ao grau de importância do item "5.1 Forma das moléculas e dos íons", observa-se que os alunos avaliaram este com média correspondente a 2,32 e os docentes com média equivalente a 2,45. Já em relação ao item "5.3 Polaridade das moléculas", os alunos avaliaram este, com média correspondente a 2,45 e os docentes com média equivalente a 2,60. Em relação aos itens "5.2 Modelo VSEPR (Valence Shell Electron Pair Repulsion)" e "5.4 Hibridização dos orbitais", estes foram classificados como "Excluir" pelos alunos e "Reavaliar" pelos docentes (Tabela 4). Verifica-se que os alunos avaliaram o item "5.2 Modelo VSEPR" com média de importância correspondente a 2,21 e os docentes avaliaram com média equivalente a 2,00. Já em relação ao item "5.4 Hibridização dos orbitais", observa-se que os alunos avaliaram este com média equivalente a 1,98 e os docentes com média correspondente a 1,80.

No entanto, os itens "5.2 Modelo VSEPR" e "5.4 Hibridização de orbitais" que correspondem uma ferramenta na previsão da geometria da molécula, e que na visão dos alunos deverão ser excluídos, provavelmente está relacionado a dificuldade do aluno de arquitetar suas ideias num espaço tridimensional.

Segundo França et al. (2012), no ensino da geometria molecular incluem-se concepções atuais e não tangíveis que exigem do discente uma habilidade perceptiva que ele ainda não adquiriu, talvez por não constituir visualização num plano tridimensional, podendo ocasionar dificuldades para o aprendizado deste e de outros conteúdos interdependentes. Além disso, estes itens possuem pouca aplicabilidade para as disciplinas específicas que o aluno vai cursar.

\subsection{Fundamentos Das Reações Químicas}

Na Tabela 5 são apresentados os cálculos relacionados à validação dos itens na dimensão "Fundamentos das reações químicas". 
Tabela 5: Resultados do método de Lawshe para a dimensão "Fundamentos das reações químicas".

\begin{tabular}{|c|c|c|c|c|c|c|c|}
\hline Item & Estrato & $\mathrm{Ne}$ & $\mathrm{N}$ & $\% \mathrm{Ne}$ & CVRcalc & CVRtab & Decisão \\
\hline \multirow{2}{*}{6.1 - Mols e Massas molares } & Alunos & 70 & 83 & $84,3 \%$ & 0,687 & 0,215 & Manter \\
\hline & Docentes & 10 & 11 & $90,9 \%$ & 0,818 & 0,591 & Manter \\
\hline \multirow{2}{*}{$\begin{array}{l}6.2 \text { - Determinação de fórmulas } \\
\text { químicas }\end{array}$} & Alunos & 65 & 80 & $81,3 \%$ & 0,625 & 0,219 & Manter \\
\hline & Docentes & 6 & 11 & $54,5 \%$ & 0,091 & 0,591 & Reavaliar \\
\hline \multirow{2}{*}{$\begin{array}{l}6.3-\text { Equações Químicas e } \\
\text { balanceamento de reações }\end{array}$} & Alunos & 70 & 83 & $84,3 \%$ & 0,687 & 0,215 & Manter \\
\hline & Docentes & 5 & 11 & $45,5 \%$ & $-0,091$ & 0,591 & Reavaliar \\
\hline \multirow{2}{*}{$\begin{array}{l}6.4-\text { Cálculos estequiométricos } \\
\text { envolvendo número de mols e massa }\end{array}$} & Alunos & 70 & 83 & $84,3 \%$ & 0,687 & 0,215 & Manter \\
\hline & Docentes & 7 & 11 & $63,6 \%$ & 0,273 & 0,591 & Reavaliar \\
\hline \multirow{2}{*}{$\begin{array}{l}6.5 \text { - Rendimento reacional, limites de } \\
\text { reação }\end{array}$} & Alunos & 48 & 71 & $67,6 \%$ & 0,352 & 0,233 & Manter \\
\hline & Docentes & 4 & 11 & $36,4 \%$ & $-0,273$ & 0,591 & Reavaliar \\
\hline
\end{tabular}

De acordo com a Tabela 5, observa-se que o único item que foi classificado como "Manter" tanto na opinião dos alunos quanto na dos docentes foi o "6.1 Mols e Massas molares". Observa-se também, que $84,3 \%$ dos alunos e 90,9\% dos docentes avaliaram este item como "Essencial". Quanto ao grau de importância, verifica-se que os alunos e docentes avaliaram este item com médias correspondentes a 2,80 e 2,82 respectivamente. É possível observar, de acordo com a Tabela 5, que itens como "6.2 Determinação de fórmulas químicas", "6.3 Equações químicas e balanceamento de reações", "6.4 Cálculos estequiométricos envolvendo número de mols e massa" e "6.5 Rendimento reacional, limites de reação" foram classificados como "Manter" pelos alunos e "Reavaliar" pelos docentes.

Tratando-se do item "6.1 Mols e Massas Molares" que deverá ser mantido tanto na percepção de alunos como na de docentes, provavelmente poderá estar relacionado a direta conexão deste item com as disciplinas específicas da Farmácia, visto que, os cálculos para a determinação do número de Mols e Massas Molares contribuem para uma adequada concentração no preparo dos medicamentos, cosméticos e preparações químicas que poderão ser utilizadas no laboratório de farmácia de acordo com a disciplina específica de Farmacotécnica.

\subsection{Misturas e Soluções}

Na Tabela 6 são apresentados os cálculos relacionados à validação dos itens na dimensão "Misturas e Soluções".

Tabela 6: Resultados do método de Lawshe para a dimensão "Misturas e Soluções".

\begin{tabular}{llllllll}
\hline Item & Estrato & $\mathrm{Ne}$ & $\mathrm{N}$ & $\% \mathrm{Ne}$ & $\mathrm{CVRcalc}$ & $\mathrm{CVRtab}$ & Decisão \\
\hline \multirow{2}{*}{7.1 - Conceitos e Classificação } & Alunos & 60 & 83 & $72,3 \%$ & 0,446 & 0,215 & Manter \\
\cline { 2 - 8 } & Docentes & 10 & 11 & $90,9 \%$ & 0,818 & 0,591 & Manter \\
\hline \multirow{2}{*}{7.2 - Técnicas de Separação } & Alunos & 60 & 81 & $74,1 \%$ & 0,481 & 0,218 & Manter \\
\cline { 2 - 8 } & Docentes & 9 & 11 & $81,8 \%$ & 0,636 & 0,591 & Manter \\
\hline \multirow{2}{*}{ Fusão } & Alunos & 59 & 81 & $72,8 \%$ & 0,457 & 0,218 & Manter \\
\hline \multirow{2}{*}{$7.4-$ Molaridade e Molalidade } & Alunos & 67 & 81 & $82,7 \%$ & 0,654 & 0,218 & Manter \\
\cline { 2 - 8 } & Docentes & 8 & 11 & $72,7 \%$ & 0,455 & 0,591 & Reavaliar \\
\hline \multirow{2}{*}{7.5 - Diluição } & Alunos & 72 & 82 & $87,8 \%$ & 0,756 & 0,216 & Manter \\
\hline \multirow{2}{*}{7.6 - Fração Molar } & Docentes & 10 & 11 & $90,9 \%$ & 0,818 & 0,591 & Manter \\
\hline \multirow{2}{*}{7.7 - Solubilidade } & Alunos & 52 & 76 & $68,4 \%$ & 0,368 & 0,225 & Manter \\
\cline { 2 - 8 } & Docentes & 6 & 11 & $54,5 \%$ & 0,091 & 0,591 & Reavaliar \\
\hline
\end{tabular}

De acordo com a Tabela 6, observa-se que tanto os alunos, quanto os docentes classificaram como "Manter" os itens: "7.1 Conceitos e Classificação", "7.2 Técnicas de 
separação", "7.3 Ponto de ebulição e ponto de fusão", "7.5 Diluição" e "7.7 Solubilidade". Verifica-se, de acordo com a Tabela 6, que os itens "7.4 Molaridade e Molalidade" e "7.6 Fração Molar" foram classificados como "Manter" pelos alunos, e "Reavaliar" pelos docentes. Também se verifica, que $80,7 \%$ dos alunos avaliaram o item "7.4 Molaridade e Molalidade" como "Essencial" e 14,5\% como "Importante, mas não essencial", enquanto que 72,7\% dos docentes avaliaram como "Essencial" e 27,3\% como "Importante, mas não essencial". Já em relação ao item "7.6 Fração Molar", 62,7\% dos alunos avaliaram o item como "Essencial" e 22,9\% como "Importante, mas não essencial", enquanto que 54,5\% dos docentes avaliaram como "Essencial" e 36,4\% como "Importante, mas não essencial".

Em relação aos itens "Conceitos e Classificação", "Técnicas de separação", "Ponto de Ebulição e Ponto de Fusão", "Diluição" e "Solubilidade" que deverão ser mantidos tanto na percepção de discentes como na de docentes, pode estar relacionado ao fato que estes itens serão utilizados nas disciplinas específicas de Farmácia, dentre elas, a Farmacotécnica, Farmacologia, Farmácia Hospitalar, Controle de Qualidade de Medicamentos e Homeopatia. O item "Ponto de Ebulição e Ponto de Fusão", por exemplo, está associado à disciplina específica de Controle de Qualidade de Medicamentos, uma vez que para detectar a pureza da matéria prima, o Ponto de Fusão corresponde a uma importante propriedade Físico-Química. Assim, o item "Diluição" será bastante abordado nas disciplinas de Homeopatia e Farmacotécnica Hospitalar para cálculos de diluições de medicamentos. Por outro lado, o item "Solubilidade" é bastante empregado na disciplina de Farmacologia por corresponder a um fator que influencia na ação do fármaco no organismo.

\subsection{Acidez e Basicidade}

Na Tabela 7 são apresentados os cálculos relacionados à validação dos itens na dimensão "Acidez e Basicidade".

Tabela 7: Resultados do método de Lawshe para a dimensão "Acidez e Basicidade".

\begin{tabular}{llllllll}
\hline Item & Estrato & $\mathrm{Ne}$ & $\mathrm{N}$ & $\% \mathrm{Ne}$ & $\mathrm{CVRcalc}$ & $\mathrm{CVRtab}$ & Decisão \\
\hline \multirow{2}{*}{8.1 - Conceitos gerais } & Alunos & 59 & 83 & $71,1 \%$ & 0,422 & 0,215 & Manter \\
\cline { 2 - 8 } & Docentes & 10 & 11 & $90,9 \%$ & 0,818 & 0,591 & Manter \\
\hline \multirow{2}{*}{8.2 - Neutralização } & Alunos & 69 & 83 & $83,1 \%$ & 0,663 & 0,215 & Manter \\
\cline { 2 - 8 } & Docentes & 10 & 11 & $90,9 \%$ & 0,818 & 0,591 & Manter \\
\hline \multirow{2}{*}{8.3 - Escala de pH } & Alunos & 71 & 83 & $85,5 \%$ & 0,711 & 0,215 & Manter \\
\cline { 2 - 8 } & Docentes & 9 & 11 & $81,8 \%$ & 0,636 & 0,591 & Manter \\
\hline \multirow{2}{*}{8.4 - Constante de acidez e basicidade } & Alunos & 67 & 82 & $81,7 \%$ & 0,634 & 0,216 & Manter \\
\cline { 2 - 8 } 8.5 - Solução tampão & Docentes & 7 & 11 & $63,6 \%$ & 0,273 & 0,591 & Reavaliar \\
& Alunos & 57 & 80 & $71,3 \%$ & 0,425 & 0,219 & Manter \\
\cline { 2 - 8 } & Docentes & 8 & 11 & $72,7 \%$ & 0,455 & 0,591 & Reavaliar \\
\hline
\end{tabular}

É possível observar, de acordo com a Tabela 7, que itens como "8.1 Conceitos gerais", "8.2 Neutralização" e "8.3 Escala de pH" foram classificados como "Manter" tanto na opinião dos alunos, quanto na dos docentes. Verifica-se que $71,1 \%$ dos alunos avaliaram o item "8.1 Conceitos gerais" como "Essencial", 22,9\% como "Importante, mas não essencial" e 6\% como "Não importante", enquanto que 90,9\% dos docentes avaliaram como "Essencial" e 9,1\% como "Não importante". Quanto ao item "8.2 Neutralização", 83,1\% dos alunos avaliaram como "Essencial", 12\% como "Importante, mas não essencial" e 4,8\% como "Não importante", enquanto que 90,9\% dos docentes avaliaram este como "Essencial" e 9,1\% como "Não importante". Em relação ao item "8.3 Escala de pH", 85,5\% dos alunos classificaram como "Essencial", 8,4\% como "Importante, mas não essencial" e 6\% como "Não importante", enquanto que $81,8 \%$ dos docentes classificaram como "Essencial", 9,1\% como "Importante, mas não essencial" e 9,1\% como "Não importante". Observa-se, de acordo com a Tabela 7, que os 
itens "8.4 Constante de acidez e basicidade" e "8.5 Solução Tampão" foram classificados como "Manter" pelos alunos e "Reavaliar" pelos docentes. Verifica-se que 80,7\% dos alunos avaliaram como "Essencial" o item "8.4 Constante de acidez e basicidade", 13,3\% como "Importante, mas não essencial" e 4,8\% como "Não importante", enquanto que $63,6 \%$ dos docentes avaliaram como "Essencial", 18,2\% como "Importante, mas não essencial" e 18,2\% como "Não importante". Em relação ao item "8.5 Solução Tampão", 68,7\% dos alunos avaliaram este como "Essencial", 24,1\% como "Importante, mas não essencial" e 3,6\% como "Não importante", enquanto que $72,7 \%$ dos docentes avaliaram como "Essencial", 18,2\% como "Importante, mas não essencial" e 9,1\% como "Não importante".

De acordo com os itens "Conceitos Gerais", "Neutralização" e "Escala de pH" avaliados como "manter" na percepção de alunos e docentes, pode se referir ao fato de que serão utilizados nas disciplinas específicas de Farmacologia e Farmacotécnica. O item "Neutralização" será utilizado na Farmacologia, por exemplo, no processo de neutralização da ação do suco gástrico proporcionado por um medicamento antiácido, promovendo uma ação local. No que diz respeito ao item "Escala de $\mathrm{pH}$ ", será aplicado nas disciplinas de Farmacologia ( $\mathrm{pH}$ sanguíneo, $\mathrm{pH}$ da lágrima), Farmacotécnica ( $\mathrm{pH}$ de formas farmacêuticas líquidas e semi-sólidas) e Controle de Qualidade de Medicamentos (Propriedade Química importante para o controle de qualidade).

\subsection{Funções Inorgânicas}

Na Tabela 8 são apresentados os cálculos relacionados à validação dos itens na dimensão "Funções Inorgânicas".

Tabela 8: Resultados do método de Lawshe para a dimensão "Funções Inorgânicas".

\begin{tabular}{llcccccc}
\hline Item & Estrato & $\mathrm{Ne}$ & $\mathrm{N}$ & $\% \mathrm{Ne}$ & CVRcalc & CVRtab & Decisão \\
\hline \multirow{2}{*}{9.1 - Ácidos } & Alunos & 73 & 82 & $89,0 \%$ & 0,780 & 0,216 & Manter \\
\cline { 2 - 8 } & Docentes & 9 & 11 & $81,8 \%$ & 0,636 & 0,591 & Manter \\
\hline \multirow{2}{*}{$9.2-$ Bases } & Alunos & 73 & 82 & $89,0 \%$ & 0,780 & 0,216 & Manter \\
\cline { 2 - 8 } & Docentes & 9 & 11 & $81,8 \%$ & 0,636 & 0,591 & Manter \\
\hline \multirow{2}{*}{9.3 - Sais } & Alunos & 72 & 82 & $87,8 \%$ & 0,756 & 0,216 & Manter \\
\cline { 2 - 8 } & Docentes & 9 & 11 & $81,8 \%$ & 0,636 & 0,591 & Manter \\
\hline \multirow{2}{*}{$9.4-$ Óxidos } & Alunos & 72 & 82 & $87,8 \%$ & 0,756 & 0,216 & Manter \\
\cline { 2 - 8 } & Docentes & 9 & 11 & $81,8 \%$ & 0,636 & 0,591 & Manter \\
\hline
\end{tabular}

Verifica-se, de acordo com a Tabela 8, que todos os itens, "9.1 Ácidos", "9.2 Bases", "9.3 Sais" e "9.4 Óxidos", foram classificados tanto pelos alunos, quanto pelos docentes como "Manter". Observa-se que $88 \%$ dos alunos e $81,8 \%$ dos docentes avaliaram os itens "9.1 Ácidos" e "9.2 Bases" como "Essencial". Em relação aos itens "9.3 Sais" e "9.4 Óxidos", verifica-se que 86,7\% dos alunos e 81,8\% dos docentes avaliaram como "Essencial". Quanto ao grau de importância, observa-se que os itens "9.1 Ácidos" e "9.2 Bases" foram avaliados pelos alunos com médias iguais correspondentes a 2,85 e avaliados pelos docentes, também com médias iguais, equivalentes a 2,73. Em relação aos itens "9.3 Sais" e "9.4 Óxidos" foram avaliados pelos alunos com médias iguais correspondentes a 2,84 e pelos docentes com médias iguais equivalentes a 2,73.

Todos os itens para a dimensão "Funções Inorgânicas" foram avaliados tanto na percepção de alunos como de docentes como "manter", talvez por serem utilizados praticamente na maioria das disciplinas específicas do curso técnico em farmácia. Os itens "Ácidos" e "Bases" correspondem às características dos medicamentos e estão diretamente correlacionados, por exemplo, com a disciplina específica de Farmacologia, sendo um dos fatores que influenciam a velocidade de absorção do fármaco. Já os itens "Sais" e "Óxidos" são empregados na disciplina específica de Farmacotécnica, por exemplo, na preparação de Soro Fisiológico (Sal) e na composição de pomadas para assadura como o Óxido de Zinco. 


\subsection{Química Orgânica}

Na Tabela 9 são apresentados os cálculos relacionados à validação dos itens na dimensão "Química Orgânica".

Tabela 9: Resultados do método de Lawshe para a dimensão "Química Orgânica".

\begin{tabular}{|c|c|c|c|c|c|c|c|}
\hline Item & Estrato & $\mathrm{Ne}$ & $\mathrm{N}$ & $\% \mathrm{Ne}$ & CVRcalc & CVRtab & Decisão \\
\hline \multirow{2}{*}{$\begin{array}{l}10.1-\text { Classificação de cadeias } \\
\text { carbônicas }\end{array}$} & Alunos & 50 & 78 & $64,1 \%$ & 0,282 & 0,222 & Manter \\
\hline & Docentes & 5 & 11 & $45,5 \%$ & $-0,091$ & 0,591 & Reavaliar \\
\hline \multirow{2}{*}{$\begin{array}{lll}10.2- & \text { Funções } & \text { orgânicas: } \\
\text { identificação } & & \end{array}$} & Alunos & 58 & 83 & $69,9 \%$ & 0,398 & 0,215 & Manter \\
\hline & Docentes & 8 & 11 & $72,7 \%$ & 0,455 & 0,591 & Reavaliar \\
\hline \multirow{2}{*}{$\begin{array}{l}10.3-\text { Nomenclatura de compostos } \\
\text { orgânicos }\end{array}$} & Alunos & 57 & 82 & $69,5 \%$ & 0,390 & 0,216 & Manter \\
\hline & Docentes & 6 & 11 & $54,5 \%$ & 0,091 & 0,591 & Reavaliar \\
\hline \multirow{2}{*}{10.4 - Reações químicas } & Alunos & 68 & 83 & $81,9 \%$ & 0,639 & 0,215 & Manter \\
\hline & Docentes & 8 & 11 & $72,7 \%$ & 0,455 & 0,591 & Reavaliar \\
\hline \multirow{2}{*}{10.5 - Isomeria } & Alunos & 33 & 59 & $55,9 \%$ & 0,119 & 0,255 & Reavaliar \\
\hline & Docentes & 5 & 11 & $45,5 \%$ & $-0,091$ & 0,591 & Reavaliar \\
\hline
\end{tabular}

De acordo com a Tabela 9, verifica-se que itens como "10.1 Classificação de cadeias carbônicas", "10.2 Funções orgânicas: identificação", "10.3 Nomenclatura de compostos orgânicos" e "10.4 Reações químicas" foram classificados como "Manter" pelos alunos e como "Reavaliar" pelos docentes. Enquanto o item "10.5 Isometria" foi classificado como "Reavaliar" tanto para os alunos, quanto para os docentes. É possível observar, de acordo com o item "10.1 Classificação de cadeias carbônicas" foi avaliado por $60,2 \%$ dos alunos como "Essencial", $32,5 \%$ como "Importante, mas não essencial" e 1,2\% como "Não importante", enquanto que 45,5\% dos docentes avaliaram como "Essencial", 36,4\% como "Importante, mas não essencial" e 18,2\% como "Não importante". Em relação ao item "10.2 Funções orgânicas: identificação" verifica-se que $69,9 \%$ dos alunos avaliaram como "Essencial"; 25,3\% como "Importante, mas não essencial" e 4,8\% como "Não importante", enquanto que $72,7 \%$ dos docentes avaliaram como "Essencial", 18,2\% como "Importante, mas não essencial" e 9,1\% como "Não importante".

Em relação ao item "10.3 Nomenclatura de compostos orgânicos" verifica-se que 68,7\% dos alunos avaliaram como "Essencial"; 26,5\% como "Importante, mas não essencial" e 3,6\% como "Não importante", enquanto que 54,5\% dos docentes avaliaram como "Essencial", 36,4\% como "Importante, mas não essencial" e 9,1\% como "Não importante". Quanto ao item "10.4 Reações químicas" observa-se que $81,9 \%$ dos alunos avaliaram como "Essencial"; $14,5 \%$ como "Importante, mas não essencial" e 3,6\% como "Não importante", enquanto que 72,7\% dos docentes avaliaram como "Essencial", 9,1\% como "Importante, mas não essencial" e 18,2\% como "Não importante". Em relação ao item "10.5 Isometria", pode-se observar que 39,8\% dos alunos avaliaram como "Essencial"; 24,1\% como "Importante, mas não essencial" e 7,2\% como "Não importante", enquanto que 45,5\% dos docentes avaliaram como "Essencial", 45,5\% como "Importante, mas não essencial" e 9,1\% como "Não importante". Na dimensão "Química Orgânica", todos os itens na percepção de docentes deverão ser reavaliados. Este resultado pode estar relacionado ao fato que, apesar da Química Orgânica corresponder uma dimensão de grande importância para a Farmácia, no curso técnico a disciplina não é muito aprofundada, sendo mais estudada pelos alunos da graduação.

\section{Conclusão}

Em relação aos resultados apresentados, os conteúdos de química disponibilizados para o curso técnico em farmácia necessitam ser revisados. De acordo com a pesquisa, o item 
"Números Quânticos" e "Orbitais Atômicos" quando analisado dentro da dimensão "Átomos" não contribui para as disciplinas específicas do curso técnico em farmácia. Por outro lado, quando este mesmo item é analisado na dimensão "Propriedades Periódicas" os docentes acreditam que deve ser excluído e os alunos preferem que seja reavaliado. Portanto, se torna necessária uma minuciosa avaliação do item para evitar acúmulo de conhecimentos isolados e proporcionar o acesso a conhecimentos químicos que permitam a contextualização com as disciplinas específicas do curso técnico. Foi possível identificar, também, na dimensão "Ligações Químicas" que os itens "Carga Formal" e "Estrutura de Ressonância" deverão ser eliminados. Já na dimensão "Moléculas" os itens "Modelo VSEPR" e "Hibridização de orbitais" não devem fazer parte do conteúdo de Química para o curso técnico em farmácia. Dessa forma, com uma possível exclusão destes itens, permitiria uma otimização do tempo e investimentos de esforços em conteúdos realmente prioritários para a formação técnica e o exercício da profissão, como por exemplo, itens relacionados às dimensões "Misturas e Soluções", "Acidez e Basicidade" e "Funções Inorgânicas" que foram avaliados como "manter".

É importante ressaltar que os itens identificados como "reavaliar" necessitam ser tratados com atenção para que a abordagem dos conceitos e dos conteúdos de química seja coerente com as disciplinas específicas da farmácia e acrescentem na formação dos técnicos em farmácia. Este trabalho poderá ser relevante para docentes e para alunos, devido à possibilidade de uma relação direta dos conteúdos de química com a realidade no ensino da farmácia, podendo proporcionar informações sobre a melhoria no ensino da química. Sendo assim, de acordo com os resultados encontrados, o trabalho poderá intervir para a melhoria dos conteúdos de química identificados, podendo proporcionar uma atualização do Projeto Pedagógico do Curso Técnico de nível médio em Farmácia.

\section{Referências}

ALMEIDA, Elba Cristina S. et al. Contextualização do ensino de química: motivando alunos de ensino médio. Centro de Ciências Exatas e da Natureza/Departamento de Química/ PROBEX, 2008)

AYRE, C.; SCALLY, A. J. Critical Values for Lawshe's Content Validity Ratio Revisiting the Original Methods of Calculation. Measurement and Evaluation in Counseling and Development, v. 47, n. 1, p. 79-86, 2014.

BAHEIRAEI, Azam et al. Psychometric properties of the adolescent health concern inventory: the persian version. Iranian journal of psychiatry, v. 8, n. 1, p. 28, 2013.

BAZARGANIPOUR, Fatemeh et al. Psychometric properties of the Iranian version of modified polycystic ovary syndrome health-related quality-of-life questionnaire. Human reproduction, v. 27, n. 9, p.2729-2736, 2012

BERGER FILHO, R. L. Educação profissional no Brasil: novos rumos. Revista Iberoamericana de Educación, Madrid, v. 20, p. 87-105, 1999.

BRASIL. Ministério da Educação. 2009 BRASIL. Disponível em: <portal.mec.gov.br/>. Acesso em: 29 nov. 2015.

BRASIL. Ministério da Educação. Orientações curriculares para o ensino médio. Ciências da natureza, matemática e suas tecnologias, v. 2, 135 p, 2006. Disponível em: <portal.mec.gov.br/seb/arquivos/pdf/book_volume_02_internet.pdf>. Acesso em: 05 jan. 2016. 
BRASIL. Uso Racional de Medicamentos - Temas Selecionados - Ministério da Saúde, Brasília/DF, 2012 - Livro página: 07

CARDOSO, S. P.; COLINVAUX, D. Explorando a motivação para estudar química. Química Nova, v. 23, n. 3, p. 401-404, 2000.

DANTIER, Rui Manuel Pinto. Validação e confiabilidade de questionário sobre transporte coletivo urbano por ônibus em Campos dos Goytacazes, RJ. 2014. 108 f. Dissertação (Mestrado) - Curso de Engenharia de Produção, Universidade Candido Mendes, Campos dos Goytacazes, 2014.

DEVRAJ, R.; WALLACE, L. S. Application of the content expert process to develop a clinically useful low-literacy Chronic Kidney Disease Self-Management Knowledge Tool (CKD-SMKT). Research in Social and Administrative Pharmacy, v. 9, n. 5, p. 633-639, 2013.

DRUM, C E.; HORNER-JOHNSON, W.; WALSH, E. S. Construction and validation of the outpatient health care usability profile (OHCUP). Disability and health journal, v. 5, n. 4, p. 292-297, 2012.

FRANÇA, E. L.; PEREIRA, M. B.; OLIVEIRA, F, P. O uso de modelos concretos e software no processo de ensino-aprendizagem de geometria molecular e arranjo espacial. XVI Encontro Nacional de Ensino de Química (XVI ENEQ) e X Encontro de Educação Química da Bahia (X Eduqui) Salvador, BA, Brasil - 17 a 20 de julho de 2012.

GHAVAMZADEH, S; KHALKHALI, H. R.; ALIZADEH, M. TV viewing, independent of physical activity and obesogenic foods, increases overweight and obesity in adolescents. Journal of Health, Population and Nutrition, Bangladesh, v. 31, n. 3, p.334-342, 2013.

GRECO, Maria Cecília Machado. O curso técnico em farmácia na ETSUS-SP: contribuições para o debate. Rio de Janeiro, 2009, 123f. Dissertação (Mestrado Ciências - Saúde publica). Escola Nacional de Saúde Pública Sergio Arouca, da Fundação Oswaldo Cruz, Rio de Janeiro.

HARIRI, Fatemeh Zahra et al. The Iranian version of the Premenstrual Symptoms Screening Tool (PSST): A validation study. Archives of Women's Mental Health, v. 16, n. 6, p.531-537, 2013.

LAWSHE, C. H. A quantitative approach to content validity. Personnel Psychology, Nova Jersey, v. 28, n. 4, p. 563-575, 1975.

LIMA, J. O. G.; LEITE, L. R. O processo de ensino e aprendizagem da disciplina de Química: o caso das escolas do ensino médio de Crateús/Ceará/Brasil. Revista Electrónica de Investigación en Educación en Ciencias, v. 7, n. 2, p. 72-85, 2012.

LOPES, S. V. L. M. et al. RELATO DE EXPERIÊNCIA: implantação do núcleo de educação permanente em Jataúba-PE. Revista da Universidade Vale do Rio Verde, v. 13, n. 1, p. 165$174,2015$. 
MAASOUMI, Raziyeh et al. The sexual quality of life-female (SQOL-F) questionnaire: translation and psychometric properties of the Iranian version. Reproductive Health, Londres, v. 10, n. 1, p. 25, 2013.

MIRANDA, Guilherme Melo. Avaliação de itens de questionário, sob a percepção de clientes de uma churrascaria em Campos dos Goytacazes, RJ. 2014. 46 f. Dissertação (Mestrado) Curso de Engenharia de Produção, Universidade Candido Mendes, Campos dos Goytacazes, 2014.

ORELLANO, E. M.; JUTAI, J. W. Cross-cultural adaptation of the Psychosocial Impact of Assistive Device Scale (PIADS) for puertorican assistive technology users. Assistive Technology, v. 25, n. 4, p.194-203, 2013.

PENNING, I. H. et al. A influência ensino-pesquisa-extensão na formação de acadêmicos do curso de Química. Encontro de Debates sobre o Ensino de Química EDQ, v. 1, n. 01, 2013.

PPC - Projeto Pedagógico de Curso Técnico de Nível Médio em Farmácia. Cód. Senac RJ 10725 Cód. DN 36. Eixo Tecnológico: Ambiente e Saúde, 2013

REEVE, B. B. et al. The first step to integrating the child's voice in adverse event reporting in oncology trials: A content validation study among pediatric oncology clinicians. Pediatric Blood and Cancer, v. 60, n. 7, p.1231-1236, 2013.

VITORINO, Fabrício de Campos et al. Sugestões de atividades experimentais para o ensino da química no ensino médio. Revista Augustus, v. 1, n. 26, p.799-810, 2009.

WILSON, F. R.; PAN, W.; SCHUMSKY, D. A. Recalculation of the Critical Values for Lawshe's Content Validity Ratio. Measurement and Evaluation in Counseling and Development, v. 45, n. 3, p. 197-210, 2012.

YU, D.T. W.; JONES, A. Y. M.; PANG, M. Y. C. Development and validation of the chinese version of the massachusetts general hospital acupuncture sensation scale: An exploratory and methodological study. Acupuncture in Medicine, v. 30, n. 3, p. 214-221, 2012. 